\title{
APPLICABILITY ANALYSIS OF CLOTH SIMULATION FILTERING ALGORITHM FOR MOBILE LIDAR POINT CLOUD
}

\author{
Shangshu Cai ${ }^{1,2}$, Wuming Zhang ${ }^{1,2 *}$, Jianbo $\mathrm{Qi}^{1,2}$, Peng Wan ${ }^{1,2}$, Jie Shao ${ }^{1,2}$, Aojie Shen ${ }^{1,2}$ \\ ${ }^{1}$ State Key Laboratory of Remote Sensing Science, Jointly Sponsored by Beijing Normal University and Institute of Remote \\ Sensing and Digital Earth of Chinese Academy of Sciences \\ ${ }^{2}$ Beijing Engineering Research Center for Global Land Remote Sensing Products, Institute of Remote Sensing Science and \\ Engineering, Faculty of Geographical Science, Beijing Normal University, Beijing 100875, China
}

KEY WORDS: LiDAR point cloud, Cloth simulation filtering, Mobile laser scanning, Ground points, Non-ground points

\begin{abstract}
:
Classifying the original point clouds into ground and non-ground points is a key step in LiDAR (light detection and ranging) data post-processing. Cloth simulation filtering (CSF) algorithm, which based on a physical process, has been validated to be an accurate, automatic and easy-to-use algorithm for airborne LiDAR point cloud. As a new technique of three-dimensional data collection, the mobile laser scanning (MLS) has been gradually applied in various fields, such as reconstruction of digital terrain models (DTM), 3D building modeling and forest inventory and management. Compared with airborne LiDAR point cloud, there are some different features (such as point density feature, distribution feature and complexity feature) for mobile LiDAR point cloud. Some filtering algorithms for airborne LiDAR data were directly used in mobile LiDAR point cloud, but it did not give satisfactory results. In this paper, we explore the ability of the CSF algorithm for mobile LiDAR point cloud. Three samples with different shape of the terrain are selected to test the performance of this algorithm, which respectively yields total errors of $0.44 \%, 0.77 \%$ and $1.20 \%$. Additionally, large area dataset is also tested to further validate the effectiveness of this algorithm, and results show that it can quickly and accurately separate point clouds into ground and non-ground points. In summary, this algorithm is efficient and reliable for mobile LiDAR point cloud.
\end{abstract}

\section{INTRODUCTION}

MLS technology can accurately and quickly acquire three-dimensional LiDAR (light detection and ranging) point cloud of earth surface. LiDAR point cloud filtering, which is to separate point clouds into ground and non-ground points, is an essential step in post-processing.

Many ground filtering algorithms have been proposed during previous decades, and these algorithms can be mainly divided into three categories (Zhang et al., 2016): slope-based methods
(Sithole, 2001; Susaki, 2012; Vosselman, 2000), mathematical morphology-based methods (Chen et al., 2007; Li, 2013; Li et al., 2014; Zhang et al., 2003) and surface-based methods (Nie et al., 2017; Pfeifer et al., 1999; Zhao et al., 2016). The aforementioned ground filtering algorithms has proven to be successful for airborne LiDAR point cloud. However, these algorithms commonly have the following problems: (1) parameters setting are complicated; (2) filtering results are usually unreliable in complex areas; (3) most of them are not open source.

\footnotetext{
${ }^{*}$ Corresponding author.

E-mail address: wumingz@bnu.edu.cn
} 
In recent years, cloth simulation filtering (CSF) algorithm is proved to be an accurate, automatic and easy-to-use algorithm for airborne LiDAR point cloud. Specifically, the accuracy of this algorithm is comparable with most of the state-of-the-art ground filtering algorithms, and its parameters are few and are easily set by the users without much experience. In addition, this algorithm has been developed a CloudCompare plugin.

Due to the different speed of travel, trajectory and scan distance of the laser scanning system, there are some differences between airborne and mobile LiDAR point clouds (Table 1), in which distribution feature plays an important role in the filtering result. There is much missing in mobile LiDAR point cloud due to occlusion of objects, which lead that the distribution feature is very unevenly. As a result, aforementioned filtering algorithm did not give satisfactory results for mobile LiDAR point cloud.

\begin{tabular}{ccc}
\hline Types & Airborne & Mobile \\
\hline $\begin{array}{c}\text { Point density } \\
\text { Distribution }\end{array}$ & Low, $<1000 / \mathrm{m}^{2}$ & High, $\geq 10000 / \mathrm{m}^{2}$ \\
feature & Evenly & Unevenly \\
Spatial & & \\
feature & 2.5-dimension & 3-dimension \\
Complexity & Complicated & Very complicated \\
feature & & \\
Building & Top information & Side information \\
feature & & \\
\hline
\end{tabular}

Table 1.Differences between airborne and mobile LiDAR point cloud

In this paper, we explored the performance of the CSF algorithm for mobile LiDAR point cloud. Three reference samples with the different characteristics of the terrain were tested.

The remainder of the paper is organized as follows. The principle of the CSF algorithm is described in Section 2. Next, the experiments are performed and the results are analyzed in Section 3. Finally, the conclusion is given in Section 4.
The method is based on the simulation of a simple physical process. Imagine a piece of soft enough cloth placed above the terrain, and then the cloth falls under the action of gravity. The final shape of the cloth is the DSM (digital surface model). In contrast, if the surface is turned upside down, and then a cloth with rigidness falls under the action of gravity, the final shape of the cloth is the DTM.

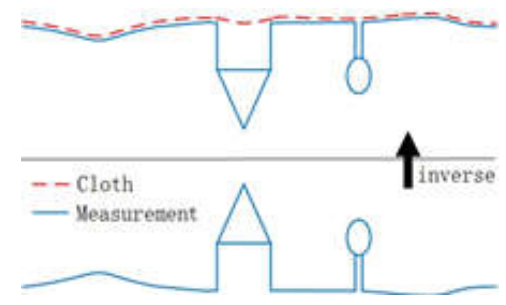

Figure 1.Overview of the CSF algorithm.

To simulate this physical process, CSF algorithm utilizes a cloth simulation technique to separate point clouds into ground and non-ground points (Zhang et al., 2016). Figure 1 illustrates the overview of this algorithm. The procedure of the algorithm is shown as follows (Figure 2):

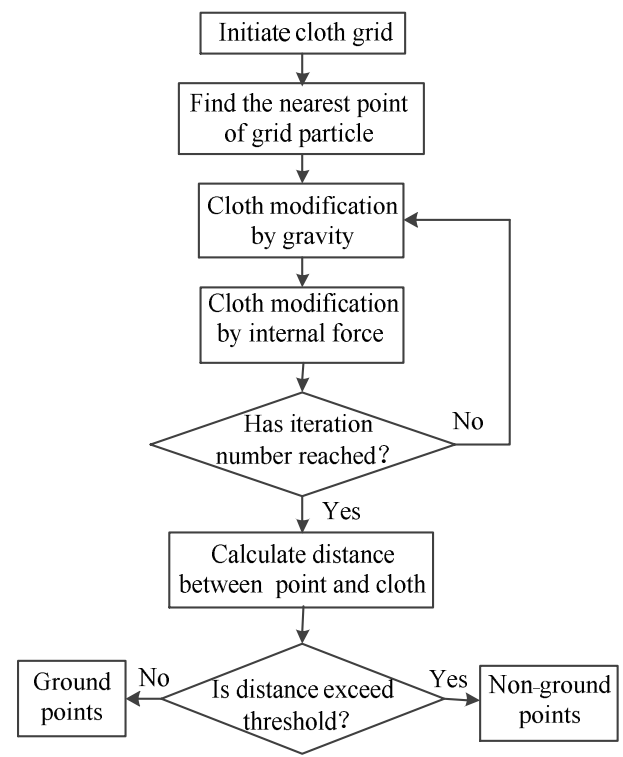

Figure 2.The flowchart of the CSF algorithm

\section{EXPERIMENTS AND RESULTS}

\section{METHOD}

Three case studies are illustrated to assess the performance of 
the CSF algorithm. As shown in Figure 3a, various non-ground objects (such as buildings, trees and poles) exist in the study area. In particular, the features of the topography for three cases are significantly different, including flat terrain, gentle slope terrain and high slope terrain. The reference datasets were obtained by manually classification, and each point was labeled as ground or non-ground points.

To verify the effectiveness of the CSF algorithm in large area, we also tested a large area dataset, which mainly include $1000 \mathrm{~m}$ road and area on both sides of road (the size is $1000 \mathrm{~m} \times 128 \mathrm{~m})$. The elevation ranges from $9 \mathrm{~m}$ to $43 \mathrm{~m}$ in the study area, whose point number is 5422298. The original dataset is shown in Figure 4a.

Four user-defined parameters are set in the CSF algorithm, including: (1) rigidness (RI), which governs the rigidness of the
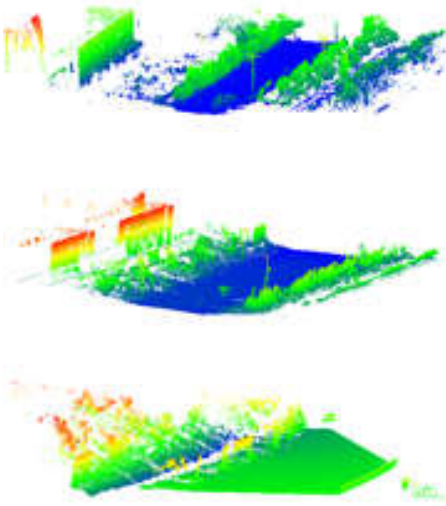

(a) cloth; (2) grid resolution (GR), which is the horizontal distance between neighboring cloth particles; (3) distance threshold (DT), which controls the final classification of all LiDAR points as ground and non-ground points based on the distance from these points to the cloth; (4) iterations numbers (IN), which controls the maximum iteration numbers. The specified parameters are listed in Table 2, in which the rigidness was set by visually inspecting the features of terrain. Other parameters were set by a few trials.

\begin{tabular}{ccccc}
\hline Samples & RI & $\begin{array}{c}\text { GR } \\
(\mathrm{m})\end{array}$ & $\begin{array}{c}\text { DT } \\
(\mathrm{m})\end{array}$ & $\begin{array}{c}\text { IN } \\
\text { (numbers) }\end{array}$ \\
\hline 1 & 3 & 0.3 & 0.15 & 150 \\
2 & 2 & 0.2 & 0.15 & 200 \\
3 & 1 & 0.3 & 0.2 & 300 \\
\hline
\end{tabular}

Table2. Parameters for each sample

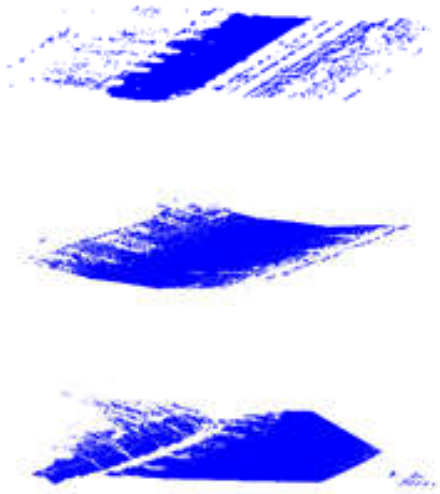

(b)

Figure 3.Results of each sample: (a) original datasets colored by height; (b) the ground points extracted from the CSF algorithm.

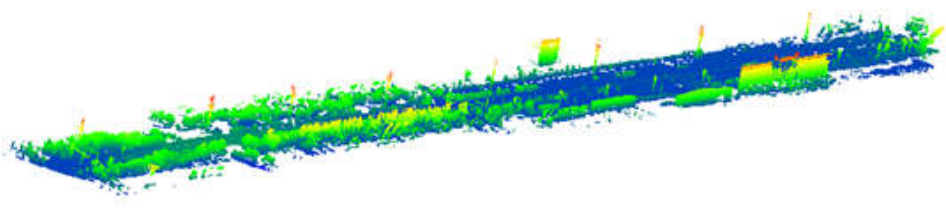

(a)

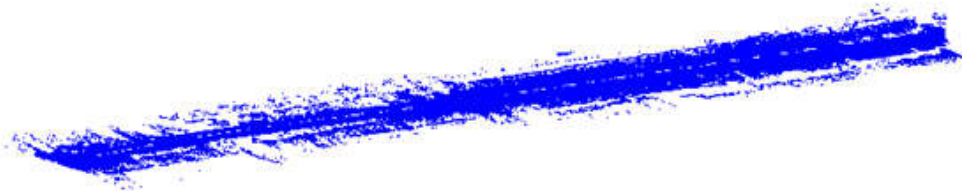

(b)

Figure 4.Result of large area dataset: (a) original dataset colored by height; (b) the ground points extracted from the CSF algorithm. 
In Figure 3b, it can be seen the CSF algorithm can filter out most of non-ground points from the original point cloud with the different features of terrain, and the terrain characteristics can be effectively remained as well.

For the large area dataset, the algorithm costed about 1.2 min to finish the experiments. By the visual inspection, we can see that this algorithm can successfully filter out non-ground points, as shown in Figure 4b.

Moreover, qualitative evaluation of the CSF algorithm was implemented by using reference samples. In this research, type I, type II and total errors were utilized to quantitatively assess the performance of this algorithm. Specifically, type I error is the rate of ground points misclassified as non-ground points, type II error is the rate of non-ground points misclassified as ground points, and the total error is the rate of misclassified points, which equation is presented as follows:

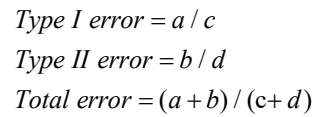

as non-ground points, $b$ represents the number of non-ground points misclassified as ground points, and $c$ and $d$ represent the total number of ground and non-ground points, respectively.

Table 3 shows the accuracy assessment of CSF algorithm, and Figure 5 shows the corresponding spatial distributions of the type I and type II errors, which indicates that this algorithm have high precision for all reference samples, and the total error is less than $1.20 \%$ for all the results. The main reason of high precision is that the simulated cloth can be directly treated as the final generated DTM for some circumstances, which avoids the interpolation of ground points, and can also recover areas of missing data. In addition, the distribution complexity of non-ground objects seldom influences the terrain approximation process (Zhang et al., 2016).

\begin{tabular}{cccc}
\hline Samples & Type I (\%) & $\begin{array}{c}\text { Type II } \\
(\%)\end{array}$ & $\begin{array}{c}\text { Total error } \\
(\%)\end{array}$ \\
\hline 1 & 0.50 & 0.37 & 0.44 \\
2 & 0.68 & 1.29 & 0.77 \\
3 & 0.94 & 2.45 & 1.20 \\
\hline
\end{tabular}

Table 3.Three types of error statistics

where $a$ represents the number of ground points misclassified
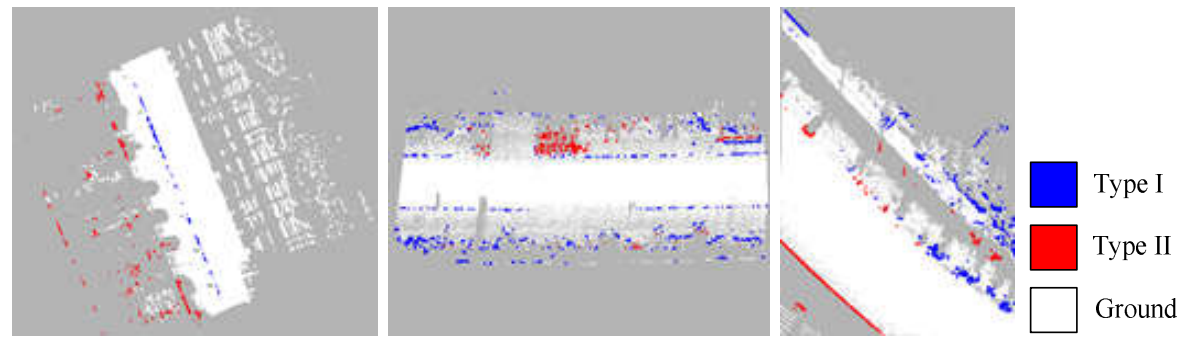

Figure 5.The spatial distributions of the type I and type II errors

\section{CONCLUSIONS}

This research explores the effectiveness of the CSF algorithm for mobile LiDAR point cloud. Three samples with different shape of the terrain are tested. Results show that this algorithm can acquire relatively high precision for all benchmark samples. Moreover, the large area dataset also is tested, and the ground points can be successfully extracted, which indicated this algorithm is universal and reliable for mobile LiDAR point cloud. This works can provide some experience for mobile LiDAR point cloud filtering processing.

\section{ACKNOWLEDGEMENTS}

This work was supported by the National Natural Science Foundation of China Grant Nos. 41331171, 41671414 and 41171265. This work was also supported by the National Basic 
Research Program of China (973 Program) Grant No. 2013CB733402. The National Key Research and Development Program of China (NO.2016YFB0501404)

\section{REFERENCES}

Chen, Q., Gong, P., Baldocchi, D., Xie, G., 2007. Filtering Airborne Laser Scanning Data with Morphological Methods. Photogramm. Eng. Remote Sens. 73, 175-185. https://doi.org/10.14358/PERS.73.2.175

Li, Y., 2013. Filtering Airborne Lidar Data By an Improved Morphological Method Based on Multi-Gradient Analysis. ISPRS - Int. Arch. Photogramm. Remote Sens. Spat. Inf. Sci. XL-1/W1, 191-194. https://doi.org/10.5194/isprsarchives-XL-1-W1-191-2013

Li, Y., Yong, B., Wu, H., An, R., Xu, H., 2014. An improved top-hat filter with sloped brim for extracting ground points from airborne lidar point clouds. Remote Sens. 6, 12885-12908. https://doi.org/10.3390/rs61212885

Nie, S., Wang, C., Dong, P., Xi, X., Luo, S., Qin, H., 2017. A revised progressive TIN densification for filtering airborne LiDAR data. Meas. J. Int. Meas. Confed. 104, 70-77. https://doi.org/10.1016/j.measurement.2017.03.007

Pfeifer, N., Reiter, T., Briese, C., Rieger, W., 1999. Interpolation of high quality ground models from laser scanner data in forested areas. Int. Arch. Photogramm. Remote Sens. 32, 31-36. https://doi.org/10.1017/CBO9781107415324.004

Sithole, G., 2001. Filtering of Laser Altimetry Data Using a Slope Adaptive Filter. Int. Arch. Photogramm. Remote Sens. Spat. Inf. Sci. XXXIV, 203-210.

Susaki, J., 2012. Adaptive slope filtering of airborne lidar data in urban areas for Digital Terrain Model (DTM) generation. Remote Sens. 4, 1804-1819. https://doi.org/10.3390/rs4061804

Vosselman, G., 2000. Slope based filtering of laser altimetry data. Int. Arch. Photogramm. Remote Sensing, Vol. 33, Part $\mathrm{B} 3 / 2$ 33 , 678-684.
Zhang, K., Chen, S.C., Whitman, D., Shyu, M.L., Yan, J., Zhang, C., 2003. A progressive morphological filter for removing nonground measurements from airborne LIDAR data. IEEE Trans. Geosci. Remote Sens. 41, 872-882. https://doi.org/10.1109/TGRS.2003.810682

Zhang, W., Qi, J., Wan, P., Wang, H., Xie, D., Wang, X., Yan, G., 2016. An easy-to-use airborne LiDAR data filtering method based on cloth simulation. Remote Sens. 8. https://doi.org/10.3390/rs8060501

Zhao, X., Guo, Q., Su, Y., Xue, B., 2016. Improved progressive TIN densification filtering algorithm for airborne LiDAR data in forested areas. ISPRS J. Photogramm. Remote Sens. 117, 79-91. https://doi.org/10.1016/j.isprsjprs.2016.03.016

https://doi.org/10.1016/S0924-2716(98)00009-4 